\title{
Descriptions of some New Plants from Eastern Asia, chiefly from the Island of Formosa, presented by Dr. Augustine Henry, F.L.S., to the Herbarium, Royal Gardens, Kew.
}

\author{
BV \\ W. BOTTING HEMSLEY, F.R.S. \\ Principal Assistant, Herbarium, Royal Gardens, Kew.
}

With Plates VII and VIII.

I N May of last year Kew received a collection of dried 1 plants from Formosa, presented by Dr. A. Henry, the same gentleman who previously collected so successfully in Hupeh and Szechuen, and who has sent to Kew probably not less than five hundred species previously unknown in the botanical world, and also a considerable number of new genera. Altogether the collection contained about 1500 species, and the following is a selection therefrom, with a few from the mainland of China. It was the wish of the Director of Kew that the whole collection should be elaborated and published, but pressure of other work has prevented this from being done, and in justice to Dr. A. Henry it was decided not to delay any longer the publication of those already described. They were partly collected by Dr. Henry himself in the neighbourhood of Takow, and at the South Cape; partly by a Chinese in the mountains of Bankinsing, in the interior of the southern peninsula; and partly by a Mr. Schmüser,

[Annals of Botany, Vol. IX. No. XXXIII. March, I895.] 
a lighthouse keeper at South Cape. Although Ape's Hill, near Takow, had been previously botanised, Dr. Henry succeeded in finding a number of interesting novelties. On a map in the Proceedings of the Royal Geographical Society, vol. vii, I 885 , the height of Ape's Hill is given as 11 Io feet, and the mountains in the vicinity of Bankinsing as 8050 feet ; but Dr. Henry's collector did not reach a greater altitude than 2000 feet, being in constant fear of the native savages. The Orchids are described by my colleague, R. A. Rolfe, A. L. S.

Hypericum (Aseyron) trinervium, Hemsl.

Frutex undique glaberrimus, ramulis teretibus. Folia conferta, subsessilia, chartacea, ovato-oblonga, $\mathbf{I}-\mathbf{I} \frac{1}{2}$ poll. longa, apice obtusa vel rotundata et simul apiculata, basi subcuneata, a basi trinervia, subtus pallidiora, crebre minuteque pellucido-punctata. Flores vix I poll. diametro, pedunculis axillaribus $3^{-6}$ lineas longis solitariis vel superioribus 2-3-floris nudis; sepala leviter inaequalia, ovata vel oblonga, obtusa $\mathbf{I}-\mathbf{I} \frac{\mathbf{1}}{4}$ lin. longa ; petala valde obliqua, apice rotundata; stamina pentadelpha, quam petala breviora; ovarium 5 -loculare, stylis per totam longitudinem connatis, stigmate parvo clavato. Capsula subcylindrica, leviter curvata, circiter semipollicaris, stylo $3^{-4}$ lineas longo coronata, demum arcte recurva; semina numerosissima, cylindrico-clavata, unilateraliter obscure alata.

Formosa: South Cape, Schmüser (Hb. Henry, 906 and 906 A).

This and the following species belong to a small group inhabiting Eastern Asia, characterized by the styles being consolidated throughout their whole length. $H$.trinervium resembles $H$. formosanum, Maxim. in foliage, but it is readily distinguished by the lateral nerves of the leaves and the much less conspicuous glands. The flowers of the latter, too, are borne on short lateral branchlets or peduncles bearing one or two pairs of leaf-like bracts. The closely reflexed ripe capsules is a striking character this has in common with H. geminiflorum. Hemsl.

Hypericum (Ascyron) geminiflorum, Hemsl.

Frutex tripedalis (fide $A$. Henry) undique glaberrimus, ramulis gracilibus rubescentibus. Folia subsessilia, tenuiter chartacea, ovatooblonga, usque ad $\frac{3}{4}$ poll. longa, apiculata, pallida, subtus glauca, obscurissime pellucido-punctata. Flores in axillis foliorum superiorum 
saepissime solitarii, vel supremi supra folia geminatim racemosi (folia cito decidua), pedunculis $3^{-6}$ lineas longis; sepala leviter inaequalia, ovato-oblonga, obtusa, circiter lineam longa; petala .... stamina .... Capsula 5-locularis, cylindrica vel teres, stylo simplice brevi coronata, 4-5 lineas longa, demum insigniter reflexa; semina minuta, cylindricoclavata.

Formosa: Ape's Hill, Takow (Hb. Henry, Ir 55).

The absence of lateral nerves traversing the whole length of the leaves, the very obscure glands, pale leaves and the absence of leaves from the upper part of the branches in the fruiting stage, distinguish this from the preceding.

Capparis membranacea, Gard. et Champ. var.? angustissima, Hemsl., foliis usque ad 8 poll. longis et maximis 3 lineas latis.

Formosa: Bankinsing mountains. White-flowered climber ( $\mathrm{Hb}$. Henry, 47 I, 1005).

This appears to be an extreme variety of a very heterophyllous species. Dr. Henry's 410 from the same locality, has somewhat broader leaves; and the specimens from China proper exhibit a considerable variety in shape, though none has them so narrow as those described above.

\section{Capparis ( $\$$ Eucapparis) formosana, Hemsl.}

Frutex alte scandens (fide $A$. Henry), ramulis floriferis crassiusculis cinereis pulverulentis. Folia distincte petiolata, coriacea, oblonga, ovato-oblonga, vel superiora minora anguste obovata vel oblanceolata, maxima cum petiolo usque ad 7 poll. longa sed saepius breviora, apice obtusissima vel rotundata atque brevissime acuminata, basi rotundata vel cuneata, integerrima, utrinque glaberrima, supra subnitida, subtus pallida, venis primariis lateralibus utrinque circiter 6-8 inter se conspicue anastomosantibus in foliis siccis utrinque sat conspicuis; petiolus 6-1o lineas longus. Flores rosei, fragrantissimi (fide Henry), circiter I poll. diametro, racemoso-fasciculati vel subumbellati cum paucis infra umbellas positi, racemi quam folia breviores, pulverulenti, pedicellis usque ad $\mathrm{I} \frac{1}{2}$ poll. longis; sepala 4 , quorum 2 exteriora pulverulenta, crassa, subcarnosa, valvata, aequalia, alte concava, orbicularia, intus nuda, 2 interiora tenuiora, petaloidea, lata, marginata, uno breviter galeato; petala lata, sepala vix aequantia; stamina numerosissima, bene evoluta non visa; ovarium glabrum, longe graciliterque 
stipitatum, placentis 3 et ovulis numerosissimis. Fructus lignosus, globosus, circiter I poll. diametro (an maturus?), stipite fere bipollicari; semina ignota.

Formosa: Bankinsing and Ape's Hill (Hb. A. Henry, 50 I, 50 I A, 5 ㅇ $\mathrm{B}, 5$ 이 $\mathrm{C}, 5$ 이 D).

Camellia gracilis, Hemsl.

Frutex ro-pedalis (fide $A$. Henry) ramulis floriferis gracillimis primum parce pilosulis cinereis. Folia brevissime petiolata, tenuia, fere membranacea, anguste lanceolata, 2-3 poll. longa, et usque ad 9 lineas lata, caudato-acuminata, simul obtusiuscula, minute apiculatoserrata, concoloria, pallide viridia, subtus secus costam primum parcissime pilosula ; petiolus circiter lineam longus, magis pilosus. Flores circiter 9 lineas diametro, pseudoterminales, erecti, solitarii, pedunculis $3-4$ lineas longis bracteis paucis parvis instructis; sepala 5 , aequalia, parva, crassa, coriacea, rotundata, pubescentia; petala 5, saepius suborbicularia, undulata, breviter unguiculata, mucronulata, extus plus minusve sericea ; stamina numerosissima, 5-6 interioris exceptis, alte monadelpha, filamentis dense barbatis, filamentis liberibus valde incrassatis clavatis; ovarium hirsutum, triloculare; stylus etiam hirsutus stamina excedens, alte trifidus. Capsula ignota.

Formosa: Bankinsing mountains (Hb. Henry, г6 г 2 ).

Closely allied to C. assimilis, Champ., and possibly a variety of it, though it has much thinner leaves, erect, smaller flowers, and exhibits other differences; all of which however may be due to local conditions and not permanent.

Actinidia lanata, Hemsl. Species ex affinitate A. fulvicomae, sed magis hirsuta, foliis late ovato-cordatis, pedunculis saepius trifloris, etc.

Frutex ramulis floriferis foliis fructibusque ferrugineo-lanatis vel tomentosis. Folia longe petiolata, crassa, chartacea, cum petiolo pollicari usque ad 6 poll. longa et $3 \frac{1}{2}$ poll. lata, longe acuminata, basi breviter cordata, aculeolato-ciliata, supra hispidulo-strigillosa, subtus stellato-tomentosa vel lanata. Pedunculi petiolos vix excedentes. Flores ... . calycis fructiferi sepala crassa, lanata, ovata, obtusa, 4-5 lineas longa.

Bacca (immatura tantum visa) villosa, ovoidea, 9-10 lineas longa, stylis numerosissimis coronata.

China: Kwangtung along the North-west river, Mr. C. Ford's native collector; 228 of 1890 collection. 
Echinocarpus sinensis, Hemsl. Species quoad fructum $E$. murici simillima sed foliis multo-majoribus oblanceolatis tenuioribus.

Arbor r 6-pedalis (A. Henry), ramulis fructiferis foliisque glaberrimis. Folia graciliter petiolata, tenuia, papyracea, anguste oblanceolata, cum petiolo $4^{-8}$ poll. longa, $\mathbf{I} \frac{1}{2}-2$ poll. lata, abrupte acutoque acuminata, deorsum attenuata sed basi obtusa, remote calloso-serrulata, venis primariis lateralibus utrinque 7-10; petiolus gracilis, 6-I 2 lineas longus, basi incrassatus, apice geniculatus. Flores ignoti. Capsula solitaria, pedicellata, pedicellis circiter sesquipollicaribus, lignosa, 4-5 locularis, globosa, I-I $\frac{1}{4}$ poll. diametro, densissime setoso-aculeata, simul tomentosa, setis rectis rigidis semipollicaribus setulosis ; semina cylindrico-oblonga, arillo ceraceo rubro ad $\frac{2}{3}$ vestita.

China: province of Hupeh, on cliffs (A. Henry, 7488).

\section{Zanthoxylum stenophyllum, Hemsl.}

Frutex $5^{-6}$ pedalis vel scandens (A. Henry), fere undique glaber, dense ramosus, ramulis floriferis fructiferisque gracilibus aculeis paucis brevibus rigidis armatis. Folia pinnata, rachi gracillima saepius elongata (interdum usque 9 poll. longa) aculeis numerosis rigidis $\mathbf{1} \frac{1}{2}-3$ lin. longis leviter curvatis instructis; foliola $5-9$, alterna vel subopposita, breviter petiolulata, subcoriacea, anguste lanceolata vel linear-lanceolata, $\mathbf{I} \frac{\mathbf{1}}{2}-3$ poll. longa, sursum longe attenuata, sed vix acuta, basi cuneata vel subrotundata, minute glanduloso-serrata, supra nitida vel subopaca, secus costam primum puberula. Cymae corymbosae vel subumbellatae, axillares, brevissime pedunculatae, pauciflorae, fructiferae circiter $\mathbf{I} \frac{\mathbf{1}}{2}$ poll. longae et latae, pedicellis fructiferis gracilibus angulatis circiter semipollicaribus. Flores unisexuales vel polygami, $q$ ignoti, $\delta$ glabri, vix 2 lineas diametro; sepala 4 , minuta, oblonga ; petala 4, ovalia, obtusa, lineam longa; stamina 4 , antheris magnis cordiformibus; ovarium rudimentarium ovoideum, glabrum. Cocci sessiles, glabri, glandulosi, oblique-ovoidei, circiter $2 \frac{1}{2}$ lin. longi; semina nigra, nitida.

China: Hsingshan, province of Hupeh (A. Henry, 6466, 6555); South Wushan, province of Szechuen (A. Henry, 5560).

Zanthoxylum micranthum, Hemsl.

Arbor usque ad 50 ped. alta sed saepius minor (A. Henry), ex affinitate $Z$. ailanthoidei, ramis floriferis aculei paucissimis rectis 
circiter 2 lineas longis armatis. Folia pinnata, 5-10 poll. longa, parce puberula, rachi gracili compresso-tereti supra angustissime canaliculata; foliola 7-II, opposita vel subopposita, distincte petiolulata, coriacea, lanceolata, 3-5 poll. longa, longe acuminata, obtusa atque emarginata, basi saepius oblique rotundata, minutissime glanduloso-dentata, undique parce pellucido-glandulosa, glabra vel cito glabrescentia, subtus pallidiora, venis primariis inconspicuis. Cymae amplissimae usque ad $\mathbf{I} 2$ poll. diametro, terminales et axillares, multiflorae, puberulae. Flores minuti, breviter pedicellati, sepalis petalis staminibusque saepius 5. Cocci maturi non visi, saepissime 3 , nudi, punctati, 2-ovulati, stigmatibus sessilibus conniventibus capitatis.

China: Ichang, Nanto and immediate neighbourhood, Hupeh (A. Henry, 2095, 4I27, 4I27, A. 4538).

This differs from $Z$. ailanthoides in the much less numerous, less conspicuous primary veins of the leaves.

\section{Zanthoxylum fraxinoides, Hemsl.}

Frutex 5-pedalis (A. Henry), Z. undulatifolio similis sed undique glaberrimus, \&c. Rami fructiferi rigidi, recti, paucispinosi, cortice nigrescente lenticellato. Folia pinnata, 4-6 poll. longa, rachi gracili inermi vel aculeis paucis minutissimis armata supra canaliculata atque angustissime bialata; foliola 5-9, opposita vel inferiora subopposita, papyracea vel demum subcoriacea, pallida, subsessilia, (terminale petiolulatum) inaequalia, deorsum saepius gradatim minora, saepius oblonga, interdum elliptica vel lanceolata, obtusa vel subacuta, $\mathbf{I}-2 \frac{1}{2}$ poll. longa, basi saepius rotundata, crenato-denticulata, in angulis dentium unipellucido-glandulosa, venis primariis lateralibus utrinque 5-9 prominulis. Cymae axillares, subsessiles, subtrichotomo-ramosae, $\mathbf{I}-\mathbf{I} \frac{\mathbf{l}}{2}$ poll. diametro, pedicellis graciliusculis nudis $2-3$ lineas longis. Flores non visi. Cocci 2-4, saepius 2, rubri (A. Henry), ovoidei, compressi, circiter $2 \frac{1}{2}$ lineas longi, grosse glandulosi, sessiles, sub insertionem producti; semina nigra, nitida.

China: Fang, province of Hupeh (A. Henry, 6903).

\section{Zanthoxylum undulatifolium, Hemsl.}

Frutex circiter Io-pedalis valde aculeatus (A. Henry), glabrescens, ramulis fructiferis graciliusculis aculeis paucis brevissimis instructis. Folia pinnata, $5^{-8}$ poll. longa, rachi gracili, angulata, puberula, inermia vel aculeis paucissimis minutis instructa; foliola $5-9$, stricte 
opposita, sessilia, vel brevissime petiolulata (terminale graciliter petiolulatum), subcoriacea, lanceolata, $\mathbf{I} \frac{1}{2}-3$ poll. longa, longe acuminata, vix acuta, basi saepius rotundata, insigniter glandulosodenticulata atque undulata, subtus pallidiora, supra hispidula. Cymae axillares, subsessiles, pauciflorae $\mathbf{I} \frac{1}{2}-2$ poll. diametro, pedicellis graciliusculis 3-6 lin. longis puberulis. Flores non visi. Cocci 2-4, parvi, oblique ovoidei, vix 2 lin. diametro, glabri, grosse glandulosi; semina nigra, nitida.

China: Nanto and mountains to the northward, province of Hupeh (A. Henry, $393^{8}$ ); South Wushan, province of Szechuen (A. Henry, 5646); above Chungking (Faber, 234).

Zanthoxylum emarginellum, Miq. in Ann. Mus. Bot. Lugd. Bat. iii. p. 22, descript. hic amplif.

Frutex speciosissimus trunco simplice (Ford $)$. Folia ampla, glabra, pinnata, fere bipedalia ; rachis glauca, teres, crassa, aculeis numerosis rectis complanatis a basi dilatata $2-5$ lin. longis armata, parte infra foliola infima circiter 2 poll. longa; foliola speciminis fordiani 27 , opposita, subsessilia, tenuia, fere membranacea, oblongo-lanceolata, maxima 5 poll. longa (superiora ac inferiora gradatim minora), sursum attenuata, apice insigniter emarginata, basi leviter obliqua, rotundata, brevissime crenato-denticulata, ad crenas et per totam laminam grosse pellucido-glandulosa. Cymae pedunculatae, circiter 2 poll. diametro, densiusculae, pedunculis bipollicaribus aculeatis, pedicellis brevibus. Flores ... Cocci sessiles, 2-3 lineas diametro, pallidi, glabri, rugulosi; semina nigra, sphaeroidea, circiter $\mathbf{I} \frac{1}{2}$ lin. diametro.-Euonymo adfinis aromatico, s. Zanthoxylum spinosissimum, Fraxini angustiore folio punctatum, Pluk. Amalth. Bot. p. 76, et Iconogr. t. 392. f. I ; Bretschneider, Early Researches into Ch. Fl. p. 72 ; Zanthoxylum sp. 13, Hemsl. in Journ. Linn. Soc. xxiii. p. 108 .

Island of Chusan, Cunningham, Hb. Sloane, vol. xciv. p. 190 in Hb. Mus. Brit.; Kelung, Formosa, C. Ford, in Hb. Hort. Kew.

\section{Zanthoxylum echinocarpum, Hemsl.}

Arbor vel frutex aculeatus, ramulis fructiferis rigidis crassiusculis puberulis. Folia imparipinnata, 7-10 poll. longa, rachi graciliuscula puberula, praecipue subtus aculeata, aculeis circiter $\mathbf{I}$ lin. longis rigidis basi incrassatis recurvis; foliola $5^{-1} \mathbf{I}$, alterna vel interdum suboppo- 
sita, breviter petiolulata, coriacea, saepius oblonga, nunc fere elliptica nunc oblanceolata, $\mathbf{I} \frac{1}{2}-4 \frac{1}{2}$ poll. longa, $\frac{3}{4}-2$ poll. lata, abrupte obtuseque caudato-acuminata, basi saepissime rotundata, utrinque secus costam primum puberula, margine minute glandulosa excepta eglandulosa, venis primariis lateralibus numerosis furcatis et inter se connexis subtus conspicuis; petioluli puberuli, I-2 lineas longi. Flores dense racemosi, subsessiles, racemis 2-3 poll. longis. Sepala 4, oblonga, obtusa, persistentia, ut videtur valvata. Petala et stamina non visa. Cocci saepissime 4, sessiles, a latere compressi, 3-4 lineas longi, aculeis demum rigidis rectis $3^{-4}$ lineas longis armati, dispermi vel abortu monospermi; semina matura non visa.

China: Ichang and immediate neighbourhood, Hupeh (A. Henry, 34 I $6 \mathrm{~B}$ and D).

\section{Zanthoxylum dimorphophyllum, Hemsl.}

Frutex 5-10 pedalis, ramis valde spinosis (A. Henry), ramulis floriferis rigidis minute puberulis inermibus vel aculeis rectis paucissimis armatis. Folia I-vel 3 -foliolata, nunc omnia I-foliolata nunc omnia 3 -foliolata et inaequalia, interdum $\mathrm{I}$ - et 3 -foliolata intermixta, petiolis $\frac{1}{2}-\mathrm{I} \frac{1}{2}$ poll. longis; foliola crassa, coriacea, subsessilia, vel plus minusve (praesertim terminale) distincte petiolulata, circumscriptione variabilia, sed saepissime ovato-oblonga vel ovato-lanceolata, I-4 poll. longa, sed saepius $\mathbf{I} \frac{1}{2}-2 \frac{1}{2}$ poll. longa, obtusa, basi rotundata, paucicrenata, glaberrima, vel primum supra secus costam puberula, supra nitida vel subopaca, tota crebre pellucido-glandulosa, glandulis majusculis. Cymae axillares, parvae, circiter 5 -7-florae, pedicellis crassiusculis hirsutis 2-4 lin. longis. Flores unisexuales vel polygami, $\delta$ non visi, o $2 \frac{1}{2}-3$ lin. diametro, apetali, vel sepala petalis simillima, 5-9, crassa, basi incrassata, angusta, fere linearia, obtusa, longitudinaliter involuta, intus pilis paucis conspersa. Carpella 2-4, saepissime 2, lepidota, stylis crassis recurvis, stigmate magno capitato; ovula 2, collateralia, pendula. Cocci globosi, circiter 2 lin. diametro; semina nigra, nitida.

China: various localities near Ichang in the province of Hupeh (A. Henry, 3902, 4462, 45 I 2, 55 I 2, 7003).

Celastrus hypoglaucus, Hemsl.

Frutex alte scandens (A. Henry) undique glaberrimus, ramulis floriferis graciliusculis, cortice rubescente vel nigrescente. Folia graciliter petiolata, papyracea vel vix coriacea, oblongo-ovata, 
elliptica vel interdum fere orbicularia, cum petiolo $2 \frac{1}{2}-5 \frac{1}{2}$ poll. longa, maxima $2 \frac{1}{2}$ poll. lata, saepius obtuse acuminata, basi rotundata, obscure denticulata, subtus nunc insigniter glauca nunc quam supra pallidiora. Flores flavi, 3-4 lineas diametro, saepius in racemos elongatos 4-9 poll. longos terminales dispositi, interdum cymosi, cymis axillaribus $3-5$-floris, pedicellis $3-9$ lineas longis; calycis lobi deltoidei, obtusissimi; petala quam sepala duplo longiora, oblonga vel fere elliptica, crispula, infra medium biauriculata et deorsum attenuata. Capsula sphaeroidea, nuda, circiter 3 lineas diametro, valvis $3^{-4}$ tenuibus crustaceis.

China: various localities near Ichang, Hupeh (A. Henry, 2837, 677I, 68I I); South Wushan, Szechuen (A. Henry, 5887).

\section{Ventilago elegans, Hemsl. (Plate VII.)}

Frutex scandens (A. Henry), dense ramosus, ramulis gracillimis flexuosis striato-angulatis puberulis, internodiis quam folia brevioribus. Folia disticha, breviter petiolata, subcoriacea, ovali-oblonga vel obovata, 6-1 2 lin. longa, apiculata, utrinque glabra, supra subnitida, venis primariis lateralibus utrinque saepissime 4 in dentes minutissimos excurrentibus, venis ultimis transversis creberrimis eximiis; petioli circiter I lin. longi, puberuli; stipulae minutae, setaceae, deciduae. Flores albi $\left(A\right.$. Henry), circiter $\mathbf{I} \frac{1}{2}$ lin. diametro, glabri, axillares, brevissime pedicellati, solitarii vel $2-3$ aggregati; calycis lobi crassi, deltoidei, vix acuti, intus carinati; petala quam sepala breviora, 3-lobulata, lobo intermedio multo minore, lateralibus complicatis stamen amplectentibus; ovarium 2-loculare, loculis I-ovulatis, stylo crasso. Fructus glabri ala anguste oblonga, circiter 9 lineas longa; semen unicum, testa membranacea, embryo intense viridis.

Formosa: Ape's Hill (Hb. A. Henry, 489).

Vitis formosana, Hemsl. Species $V$. umbellatae similis sed cymis compositis brevissime pedunculatis.

Frutex alte scandens (A. Henry), omnino glaber, ramulis floriferis graciliusculis angulatis, internodiis quam folia saepe brevioribus. Folia trifoliolata, breviter petiolata; foliola brevissime petiolulata, subcarnosa, inaequalia (lateralia minora plus minusve obliqua), oblonga, anguste lanceolata vel interdum fere ovata, $\mathbf{I}-2 \frac{1}{2}$ poll. longa, subacuta, obtusa vel rotundata, basi saepe cuneata, interdum rotundata, obscure paucidentata, venis subtus (in foliis siccis) prominulis ; petiolus $\frac{1}{2}-\mathbf{I}$ poll. 
longus. Cymae axillares, compositae, umbelliformes, densae, brevissime pedunculatae, circiter I poll. diametro, pedicellis $\mathbf{I}-2$ lin. longis gracilibus. Sepala minuta, dentiformia; petala ovato-oblonga, vix acuta, vix I lin. longa, patentia, demum reflexa. Bacca ignota.

Formosa: Takow, Playfair, 203 ; same locality (Hb. Henry, 745).

\section{Desmodium ( $\$$ Eudesmodium) gracillimum, Hemsl.}

Herba vel suffrutex debilis, ramulis elongatis fere filiformibus radicantibus parce albo-strigillosis. Folia unifoliolata, longe graciliterque petiolata, tenuissima, pallide viridia, cordiformia, absque petiolo $\frac{1}{2}-\mathbf{I} \frac{\mathbf{1}}{4}$ poll. longa, obtusa vel rotundata, utrinque parcissime strigillosa; petiolus gracillimus, 4-9 lin. longus; stipulae ac stipellae minutae, setuliformes. Flores perfecti non visi, parvi (circiter 2 lin. longi), albo-caerulei (Henry) pauci, in racemos laxos terminales dispositi. Legumen maturum non visum, breviter stipitatum, pubescens, angustum, rectum, circiter pollicare, articulis circiter 4 oblongis.

Formosa: Summit of Ape's Hill, Takow (Hb. Henry, I I6o).

Crotalaria similis, Hemsl. Species $C$. perpusillae, Coll. et Hemsl. (Journ. Linn. Soc. xxviii. p. 37. t. 5) simillima sed foliis angustioribus \&c.

Herba ramosissima, procumbens, undique dense longeque strigillosovillosa, ramis gracilibus $2-4$ poll. longis. Folia simplicia, subdisticha, conferta, brevissime petiolata, papyracea, ovato-oblonga vel obovata, $2 \frac{1}{2}-3$ lin. longa, apiculata, supra demum glabrescentia, punctulata. Flores pauci, ad apices ramorum conferti, breviter pedicellati, 3-4 lin. longi, bracteolis minutis lanceolatis calycis tubo adnatis; caly $x$ dense villosus, distincte bilabiatus, labio antico alte trilobo, lobis angustis acutissimis declinato-curvatis, labio postico erecto vix ad medium bifido lobis latis apice oblique rotundatis; petala calycem vix excedentia, brevissime unguiculata, striata, vexillo orbiculari circiter 3 lin. diametro extus medio apicem versus strigilloso, alis late oblongis apice rotundatis, carina e basi lata curvato-acuminata; ovarium glabrum, circiter I 2-ovulatum, stylo curvato gracili. Legumen oblongum, 4-5 lin. longum et $2-2 \frac{1}{2}$ lin. latum, oligospermum; semina parva, nitida, compressa, reniformia.

Formosa: South Cape, Schmüser (Hb. Henry, 252).

Prunus ( $\S$ Laurocerasus) xerocarpa, Hemsl.

Arbor ro-pedalis (A. Henry) fere omnino glabra, ramulis floriferis 
gracilibus primum minute puberulis vel pulverulentis rubescentibus. Folia petiolata, ut videtur persistentia, subcoriacea, lanceolata, cum petiolo 3-5 poll. longa, caudato-acuminata, obtusa, basi saepe subcuneata, integerrima, subtus pallidiora, rubro-punctata; stipulae cito deciduae non visae. Flores albi, circiter 4 lineas diametro, simpliciter racemosi vel racemoso-paniculati, paniculis folia aequantibus vel excedentibus, ramulis pedicellisque gracillimis; calycis tubus intus villosus; calycis lobi minuti, petaloidei, rotundati, crispulati ; petala fere orbicularia, circiter $\mathbf{I} \frac{1}{2}$ lin. diametro, crispulata; stamina circa 20 ; ovarium glabrum. Fructus exsuccus, pisiformis, globosus, 3-4 lin. diametro, epicarpio ut videtur tenuissimo, endocarpio crustaceo.

Formosa: Bankinsing mountains (Hb. Henry, $\mathbf{1}_{6}^{6}{ }^{6}, \mathbf{1} 65^{8}$, I $\left.65^{8}, \mathrm{~A}\right)$.

Photinia (§ Eriobotrya) deflexa, Hemsl. Eriobotryae Hookerianae proxime affinis, sed foliis longe petiolatis insigniter deflexis et grosse serratis.

Arbor 30-pedalis (A. Henry) ramulis fructiferis crassis puberulis. Folia longe petiolata, crassa, coriacea, deflexa, oblongo-lanceolata, pedalia, grosse irregulariterque serrata, serris curvatis obtusis, utrinque glabra, venis primariis lateralibus numerosis utrinque conspicuis in serras excurrentibus; petiolus $\mathbf{I} \frac{1}{2}-2 \frac{1}{2}$ poll. longus, basi incrassatus ; stipulae deciduae non visae. Inflorescentia terminalis, cymoso-corymbosa, ramulis crassis, pedicellis brevissimis. Flores ignoti. Fructus ovoideus, 6-8 lin. longus, lignoso-suberosus, calycis lobis oblongis obtusis lanatis coronatus ; semina ovoidea.

Formosa: Bankinsing (Hb. A. Henry, 498).

Itea parviflora, Hemsl.

Frutex glaber vel glabrescens, ramulis floriferis gracilibus, internodiis quam folia multo brevioribus. Folia distincte petiolata, tenuia, papyracea, glabra, anguste lanceolata vel oblongo-lanceolata, 2-4 poll. longa, acuminata, obtusa simul apiculata, basi cuneata, sursum plus minusve crenato-serrulata et subundulata, subtus pallidiora, venis primariis lateralibus utrinque saepius 5 vel 6 arcuatis subtus sat conspicuis, venis ultimis transversis pulchre reticulatis; petiolus gracilis, 3-6 lin. longus. Racemi axillares, gracillimi, $\mathbf{1}-3$ poll. longi, multiflori, interdum puberuli, pedicellis gracillimis $\mathbf{I - 2}$ lin. longis. Flores albi, circiter lineam longi, conferti; calycis dentes elongato- 
deltoidei, vix acuti, quam petala dimidio breviores, tubo intus hirtello; petala anguste ovato-lanceolata, vix acuta, medio indistincte carinata, persistentia. Capsula 2-3 lin. longa, saepius puberula, polysperma; semina elongata, gracilia, fusiformia.

Formosa: South Cape, Schmüser (Hb. A. Henry, 965, 1263, I 322 ).

Most nearly related to $I$. chinensis, differing in its narrow, thin leaves, smaller flowers, relatively broad petals, and puberulous capsule.

\section{Diospyros utilis, Hemsl.}

Arbor magna (A. Henry), ramulis fructiferis crassis sericeo-hirsutis. Folia alterna, breviter petiolata, crassa, coriacea, oblonga vel lanceolata, $5^{-8}$ poll. longa, maxima 3 poll. lata, acute acuminata, basi biauriculata vel superiora minora interdum rotundata vel cuneata, undulata, supra glabra, costa impressa, subtus albida, dense arcte appresseque sericeostrigillosa, costa elevata; petiolus crassus, teres, 3-4 lin. longus. Flores .... Fructus edulis (A. Henry) subsessilis, depresso-globosus, fere 2 poll. diametro, tomentosus, saepius 8-locularis, sepalis amplis crassis sericeo-tomentosis fere orbicularibus persistentibus subtendus ; semina oblonga, compressa, testa nigra nitida crassissima dura, albumine non ruminato.

Formosa: Bankinsing mountains (Hb. Henry, 81 5 ).

Dr. Henry describes this as a large tree furnishing a good wood, used for making axles, and an edible fruit called mao-shih, that is, hairy persimmon.

Rehmannia Oldhami, Hemsl. in Journ. Linn. Soc. xxvi, p. I94; descriptio hic amplificata.

Perenne, undique albo-hirsutum, caulibus saepius simplicibus gracilibus maximis cum inflorescentia 3 -pedalibus sed saepius brevioribus. Folia distincte petiolata, membranacea, opposita, subopposita, vel inferiora saepe alterna, saepe obliqua, ovata vel obovata, maxima cum petiolo 9 poll. longa, sed saepius breviora, grosse et inaequaliter serrata, utrinque strigilloso-hirsuta. Racemi simplices vel interdum ramosi saepe valde elongati et gracillimi, supra medium proliferi, corporibus minutis carnosis fasciculatis instructi; pedicelli demum circiter semi-pollicares. Flores flavi (A. Henry), maculati, $\mathbf{I} \frac{1}{4}-\mathbf{I} \frac{1}{2}$ poll. longi; calycis segmenta lineari-lanceolata, acutissima, 4-5 lin. 
Jonga; corolla anguste ventricoso-tubulosa, limbo obliquo lobis brevibus rotundatis; stamina 4, inclusa, antheris per paria cohaerentibus. Capsula ovoidea, calyce inclusa, in valvas 4 placenticide dehiscens; semina minuta linearia vel cylindrica, breviter funiculata, in utroque termino cristata.

Formosa: Ape's Hill, Takow, on the sides of cliffs in dry, shaded situations (Hb. A. Henry, I $05^{2}$ ).

There is now no doubt that this plant belongs to the genus Rehmannia; and there is also little doubt that this genus would be better placed in the Cyrtandreae.

\section{Mesona procumbens, Hemsl. (Plate VII.)}

Herba procumbens, radicans, caulibus elongatis 2-3-pedalibus graciliusculis pilis paucis longis debilibus instructis vel demum glabrescentibus. Folia distincte petiolata, tenuia, fere membranacea, ovata vel ovato-lanceolata, cum petiolo gracili I-2 poll. longa, acuta, basi rotundata vel subcuneata, grossiuscule calloso-serrata, praecipue subtus pilis paucis tenuissimis conspersa, venis primariis lateralibus utrinque saepius 6 ; petiolus $3^{-6}$ lineas longus. Verticillastri dense racemosi, racemis densis terminalibus vel axillaribus cum pedunculo 2-3-pollicaribus, pedicellis brevissimis; bracteae rotundatae, ciliatae, subito caudato-acuminatae, cauda demum sub laminam arcte recurva. Flores minuti, cum staminibus exsertis circiter 2 lineas longi, setulosi; caly $x$ fructifer auctus, circiter $\mathbf{I} \frac{1}{2}$ lin. longus, scariosus, longitudinaliter I0-costatus et insigniter transverse multicostatus, irregulariter bilabiatus, demum clausus, tubo leviter inflato, labio antico oblongo integro apice rotundato, labio postico inaequaliter tridentato dentibus omnibus acutis; corolla alte bilabiata, hians, tubo brevissimo, labio antico concavo cymbiformi extus parce villoso, labio postico lato breviter tridentato, dente intermedio obscure emarginato; stamina longe exserta, declinata, filamentis staminum duorum posticorum basi appendiculatis, appendiculis oblongis hirsutis. Nuculae anguste ellipsoideae, glabrae.

Formosa: South Cape: collected by Schmüser (Hb. Henry, I 3 I 7 ).

This is a very distinct species, and markedly so in its much elongated procumbent stems. It seems evident that Wallich's genus Geniosporum must be reduced to Mesona; the only difference given being the presence or absence of appendages at the base of the posterior filaments; but these are often very minute and easily 
overlooked where they do occur, and scarcely furnish a character of generic importance. Comparing our figure of Mesona procumbens with that of Geniosporum strobiliferum, Wall. in Hooker's 'Icones Plantarum' t. 462 , the resemblances, it will be seen, are very strong.

Helicia formosana, Hemsl. in Journ. Linn. Soc. xxvi, p. 394. Descriptio hic amplificata (Plate VIII).

Folia maxima fere pedalia et $4 \frac{1}{2}$ poll. lata, interdum grosse serratodentata. Racemi circiter 6 poll. longi. Fructus depresso-globosus, $\mathbf{I}-\mathbf{I} \frac{\mathbf{1}}{4}$ diametro: semen unicum, cotyledonibus hemisphaericis.

Formosa: Bankinsing mountains, near watercourses (Hb. Henry, $805)$.

Liparis macrantha, Rolfe.

Caulis erectus, subcarnosus, subelongatus. Folia membranacea, petiolata ; limbus elliptico-ovatus, breviter acuminatus, basi obliquus, $4-4 \frac{1}{2}$ poll. longus, $2-2 \frac{1}{2}$ poll. latus; petiolus latus, $I \frac{1}{4}-I \frac{3}{4}$ poll. longus, basi laxe tubuloso-vaginatus, membranaceus. Scapus $\frac{3}{4}-\mathbf{I}$ ped. altus, multiflorus. Bracteae parvae, triangulo-ovatae, acutae, reflexae, vix I lin. longae. Pedicelli 9 lin. longi. Sepalum posticum lanceolatum; lateralia falcato-lanceolata, subobtusa, $5 \frac{1}{2}-6$ lin. longa, $\mathbf{I} \frac{1}{2}$ lin. lata. Petala angustissima, 7 lin. longa. Labellum obcordatum, apiculatum, denticulatum, $5 \frac{1}{2}$ lin. longum, 5 lin. latum, basi bituberculatum. Columna $2 \frac{1}{2}$ in. longa.

Formosa: Tamsui (Hb. A. Henry, r 695 !).

A remarkable species with dark purple flowers an inch in diameter.

Phreatia formosana, Rolfe.

Planta caespitosa. Folia linearia obtusa, basi paullo attenuata, 3-6 poll. longa, 2-3 lin. lata. Scapi graciles, elongati, multiflorae, 6-7 poll. longae. Bracteae lanceolatae, acutae, $\mathbf{I}-\mathbf{I} \frac{\mathbf{1}}{2}$ lin. longae. Pedicelli $\frac{3}{4}$ lin. longi. Sepala ovata, subacuta, $\frac{3}{4}$ lin. longa. Petala ovata, subacuta, sepalis paullo minora. Labellum breviter unguiculatum; limbus late trulliformi-cordatus, subobtusus, $\frac{2}{3}$ lin. latus. Columna brevissima.

Formosa: South Cape (Hb. A. Henry, I 349).

Allied to $P$. elegans, Lindl., but the lip more distinctly trulliform. 
Agrostophyllum formosanum, Rolfe.

Caules erecti, 9-1o poll. longi, subcompressi, distichophylli.

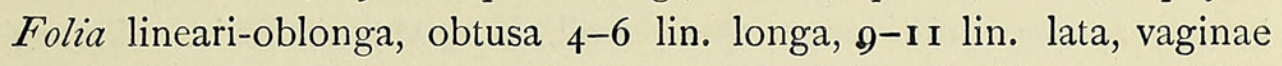
conduplicatae, imbricatae. Capitulum subsessile, densiflorum, I 6 lin. latum. Bracteae lancéolato-oblongae, obtusae, striatae, 2 lin. longae. Sepala ovata, subacuta, lateralia carinata, concava, $\mathbf{I} \frac{3}{4}$ lin. longa. Petala linearia, obtusa, torta, $\mathbf{I} \frac{3}{4}$ lin. longa. Labellum 2 lin. longum, hypochilio subhemisphaerico-saccato, epichilio reniformi-ovato obtuso. Columna crassa, I lin. longa.

Formosa: South Cape (Hb. A. Henry, I 350).

Allied to Agrostophyllum majus, Hook. f., from Perak.

Calanthe formosana, Rolfe.

Folia petiolata, lanceolata, acuta, I 2-I 4 poll. longa, $\mathbf{I} \frac{1}{2}-2 \frac{1}{4}$ poll. lata; petiolus 6-7 poll. longus. Scapus го-I2 poll. altus; vaginae spathaceae, basi tubulosae, $\mathbf{I} \frac{\mathbf{1}}{4}-\mathbf{I} \frac{3}{4}$ poll. longae. Bracteae linearilanceolatae, acutae, I-I $\frac{1}{2}$ poll. longae, $2 \frac{1}{2}-3$ lin. latae. Sepala lanceolata, acuminata, 5 lin. longa, $\mathbf{1} \frac{3}{4}-2$ lin. lata. Petala lanceolata, breviter acuminata, subobliqua, uninervia, membranacea, $4 \frac{1}{2} \operatorname{lin}$. longa, $\mathbf{1} \frac{3}{4}$ lin. lata. Labellum supra medium columnae adnatum, trilobum, 3 lin. longum, lobis lateralibus rotundatis, intermedio oblongo apiculato, disco laevi, calcare gracili, $2 \frac{1}{2}$ lin. longo. Columna 2 lin. longa, apice dilatata.

Formosa: South Cape (Hb. A. Henry, I 347).

Allied to the Indian $C$. clavata, Lindl., but with a differently shaped lip, and the spur not clavate. The bracts much resemble those of C. densiflora, Lindl., belonging to another group. The buds are not expanded, and probably the flowers exceed the dimensions here given.

Geodorum formosanum, Rolfe.

Folia oblongo-lanceolata, acuta, $\frac{1}{2}-\mathbf{I}$ ped. longa, $\mathbf{I} \frac{\mathbf{1}}{\mathbf{4}}-2 \frac{1}{2}$ poll. lata. Scapi 6-ro poll. longi, apice nutante, basi laxe vaginati. Bracteae lineari-lanceolatae, 5-6 lin. longae. Pedicelli graciles $2-2 \frac{1}{2}$ lin. longae. Sepala et petala oblonga vel spathulato-oblonga, obtusa, $4 \frac{1}{2}$ lin. longa. Labellum ovato-oblongum, obtusum, obscure bilobum, basi saccatum, 5 lin. longum, $3 \frac{1}{2}$ lin. latum, disco medio incrassato obscure tricarinato. Columna clavata, 2 lin. longa.

Formosa: Takow (Hb. A. Henry, I I 37), South Cape (Hb. A. Henry, I375). I am unable to identify this with any Indian species. 
Tropidia formosana, Rolfe.

Herba erecta, I ped. alta. Folia late lanceolata, acuta, membranacea, $4-5$ poll. longa, $\frac{3}{4}-\mathbf{I} \frac{1}{4}$ poll. lata, vaginis laxis. Racemi abbreviati, subcapitati, 6-7 lin. lati. Bracteae lanceolatae, acutae, striatae, 2 lin. longae. Pedicelli $\mathbf{I} \frac{1}{2}$ lin. longi. Sepala oblonga, acuta, $3-3 \frac{1}{2}$ lin. longa, I lin. lata, mento saccato. Petala oblongolanceolata, subobtusa, sepalis subaequalia, nervo medio incrassata. Labellum oblongum, subobtusum, basi saccatum, sepalis subaequale. Columna clavata, $\mathbf{I} \frac{1}{2}$ lin. longa.

Formosa: Bankinsing (Hb. A. Henry, I 573).

Allied to the Philippine $T$. septemnervis, Rchb. f.

\section{Zeuxine formosana, Rolfe.}

Caules subrepentes. Folia petiolata, ovata, apiculata, limbus $\mathbf{I} \frac{\mathbf{1}}{4}-\mathbf{I} \frac{3}{4}$ poll. longa, $6-\mathbf{I} \mathbf{I}$ lin. lata; petiolus 6 lin. longus, infra medium tubuloso-vaginatus. Scapus ro-r 4 poll. altus, pubescens. Bracteae ovato-lanceolatae, acuminatae, 4-5 lin. longae, pubescentes. Sepalum posticum ovatum, subobtusum, concavum, $2 \frac{1}{2}$ lin. longum ; lateralia oblonga, subobtusa, 3 lin. longa. Petala oblique semiovata, subobtusa, $2 \frac{1}{2}$ lin. longa. Labellum 3 lin. longum, basi cymbiforme, bicallosum, medio constrictum, angustum, apice dilatato-bilobum, lobis suborbicularibus. Columna lata, apice bibrachiata, $\mathbf{I} \frac{1}{2}$ lin. longa.

Formosa: South Cape (Hb. A. Henry, 644).

This species somewhat resembles Zeuxine flava, Benth., in habit, but the flowers are fully twice as large.

Cheirostylis chinensis, Rolfe.

Rhizoma repens. Folia breviter petiolata, late ovata, acuta vel apiculata, puberula, 5-8 lin. longa, $3^{-6}$ lin. lata; petiolus $2-2 \frac{1}{2} \operatorname{lin}$. longus, basi dilatatus, breviter tubuloso-vaginatus. Scapus gracilis, $3^{-5}$ poll. altus, pubescens, paucivaginatus, pauciflorus. Bracteae ellipticooblongae, obtusae, submembranaceae, $\mathbf{I} \frac{1}{2}$ lin. longae. Perianthium subhemisphaericum, $\mathbf{I} \frac{\mathbf{1}}{2}$ lin. longum. Petala elliptico-oblonga. Labellum unguiculatum, limbo flabellato bifido grosse dentato 3 lin. lato. Columna brevis, appendicibus subulatis.

Formosa: South of island (A. Hance, 390), Summit of Ape's Hill, Takow (Hb. A. Henry, 320 ); Hongkong (Ford, I 30 ).

Allied to the Indian C. flabellata, Wight. 
Goodyera formosana, Rolfe.

Herba elata, $2 \frac{1}{2}-3$ ped. alta. Folia elliptico-lanceolata vel oblonga, acuta vel subacuminata, $6-7$ poll. longa, $2-2 \frac{1}{2}$ poll. lata. Scapus pubescens, vaginis lanceolatis, numerosis. Racemus elongatus, multiflorus, I ped. longus. Bracteae lanceolatae, acuminatae, 3-5 lin.

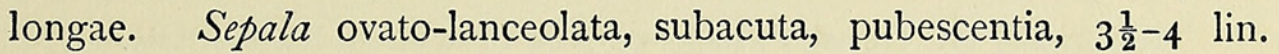
longa. Petala spathulato-lanceolata, subacuta, $3 \frac{1}{2}-4$ lin. longa. Labellum 3-3 $\frac{1}{2}$ lin. longum, basi concavo-saccatum, venis fimbriatovillosis, medio abrupte contractum, dein lineari-subulatum, apice reflexum. Columna clavata, 2 lin. longa.

Formosa: Bankinsing mountains (Hb. A. Henry, 409). 'Flowers whitish.'

This species has the habit of G. fumata, Thw., of Ceylon, to which it is most nearly allied. 


\section{EXPLANATION OF THE FIGURES IN PLATES VII AND VIII.}

Illustrating Mr. Hemsley's paper on New Plants from Eastern Asia.

\section{PLATE VII.}

A. Flowering and fruiting branches of Ventilago elegans. Natural size. Fig. I. A leaf, slightly enlarged to show the transverse venation.

Fig. 2. Vertical section of a flower.

Fig. 3. A petal spread open and stamen.

Fig. 4. The same in its natural position with the stamen drawn forward.

Fig. 5. Front view of an anther.

Fig. 6. Back view of an anther.

Fig. 7. A fruit.

Fig. 8. Vertical section of the same.

Fig. 9. Embryo, showing the large, almost terete cotyledons.

All the figures enlarged except $A$.

$B$. Flowering and fruiting stem of Mesona procumbens. Natural size.

Fig. Io. A verticillaster, with bract; all the flowers except one removed.

Fig. I1. A corolla laid open, showing the appendaged filaments.

Fig. I2. Back view of an anther.

Fig. I3. Pistil.

Fig. 14. Calyx in the fruiting stage.

Fig. I5. Fruit.

All the figures enlarged, except $B$.

\section{PLATE VIII.}

C. Flowering branch of Helicia formosana. Natural size.

Fig. I6. An expanded flower.

Fig. I 7. Upper part of a segment of the perianth with stamen attached.

Fig. 18. Back view of anther.

Fig. 19. Pistil and disk; upper part of style removed.

Fig. 20. Vertical section of ovary.

Fig. 21. A fruit.

Fig. 22. A seed from which the membranous outer testa has begun to disappear.

Fig. 23. The same wholly removed, revealing the dimorphic inner testa, the upper half of which is rugose, the lower smooth.

Fig. 24. One of the hemispherical cotyledons and minute radicle.

Figures 16 to 20 enlarged; 21 to 24 natural size. 


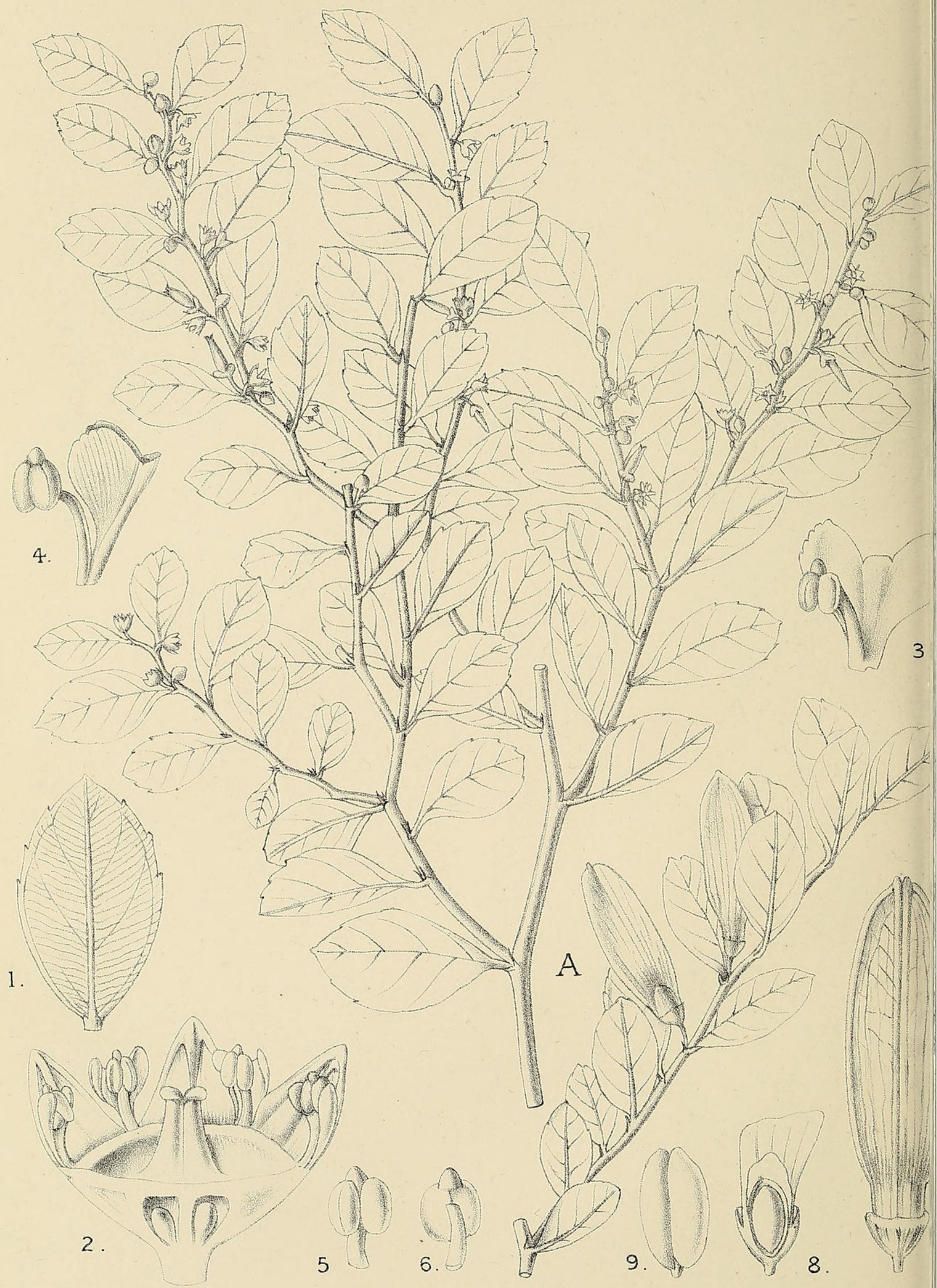

M. Smith del.

HEMSLEY. - FORMOSAN PLANTS. 
VoL.IX, PL.VII.

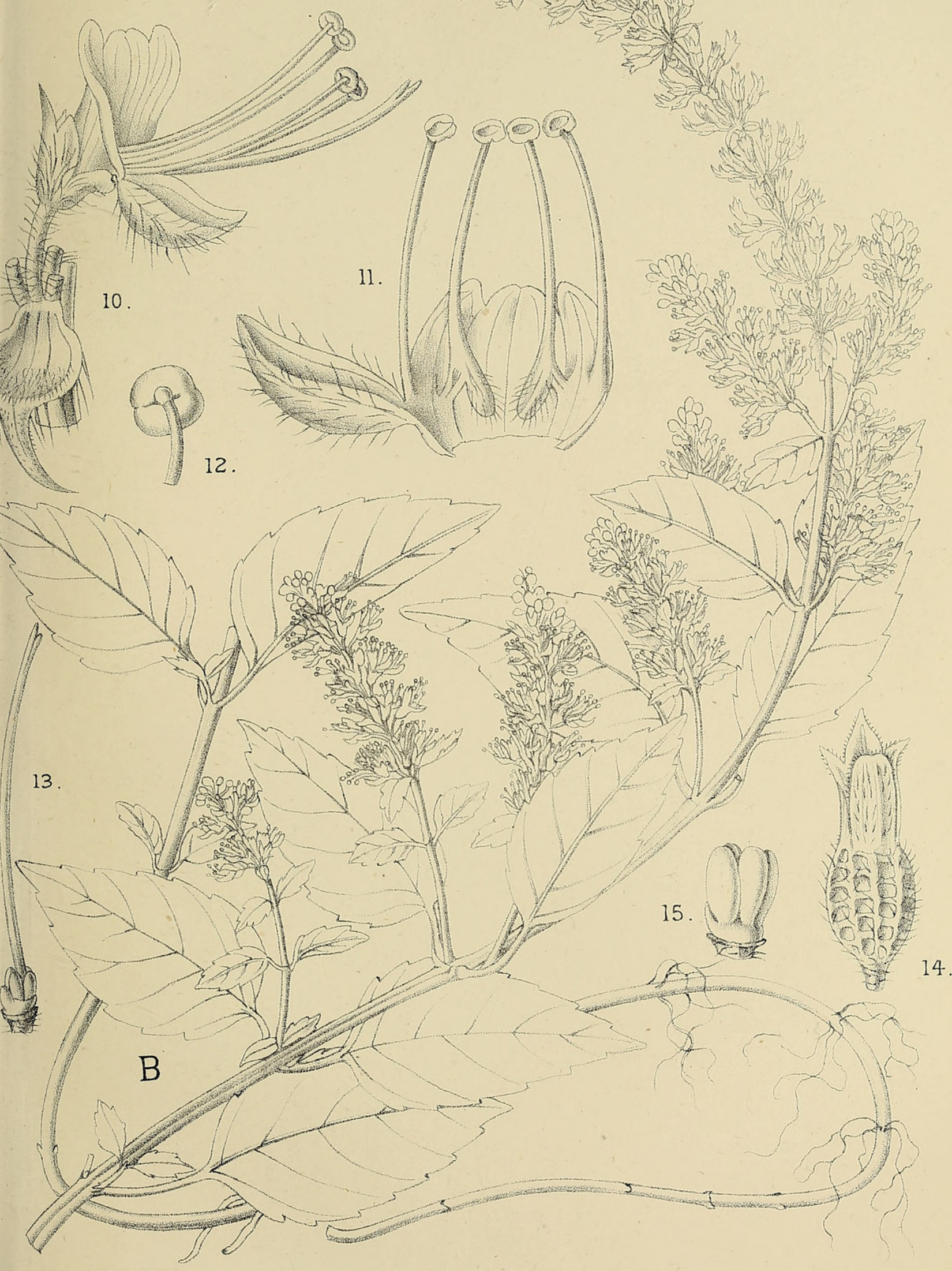

University Press, Oxford. 

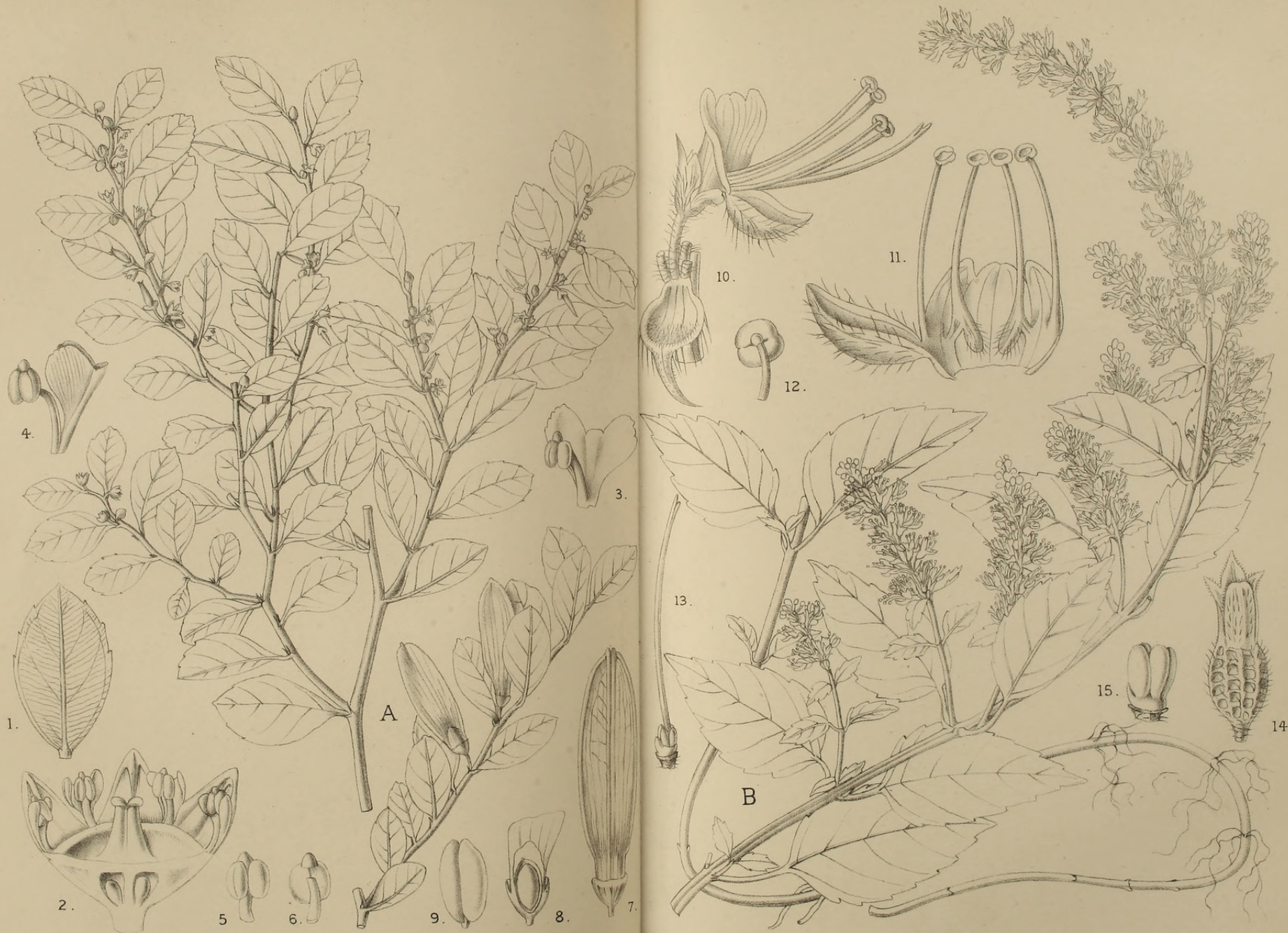


\section{Annals of Botany}

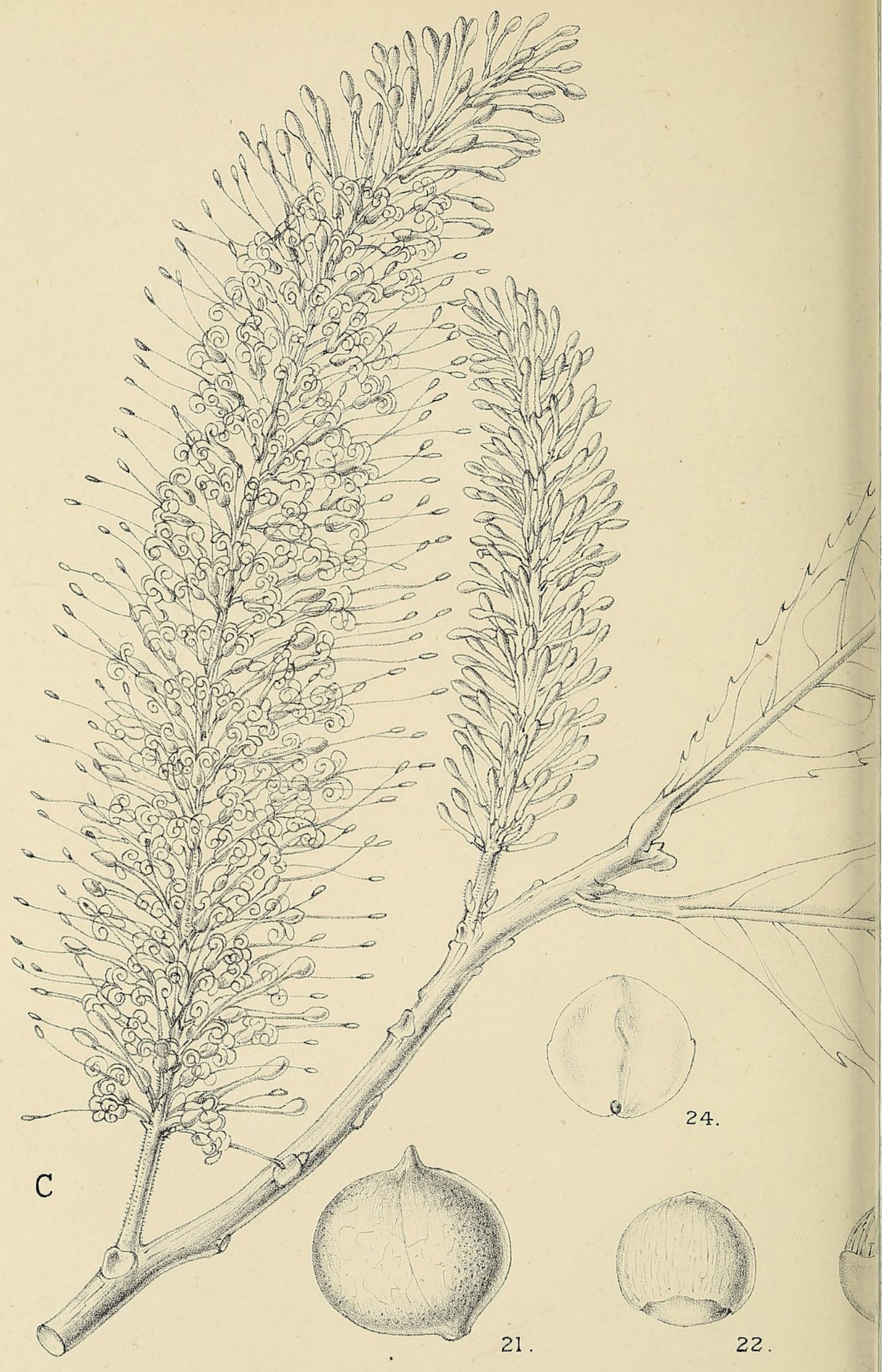

M. Smith del.

HEMSLEY. - FORMOSAN PLANTS. 
Vol.LX, PL.VIII.

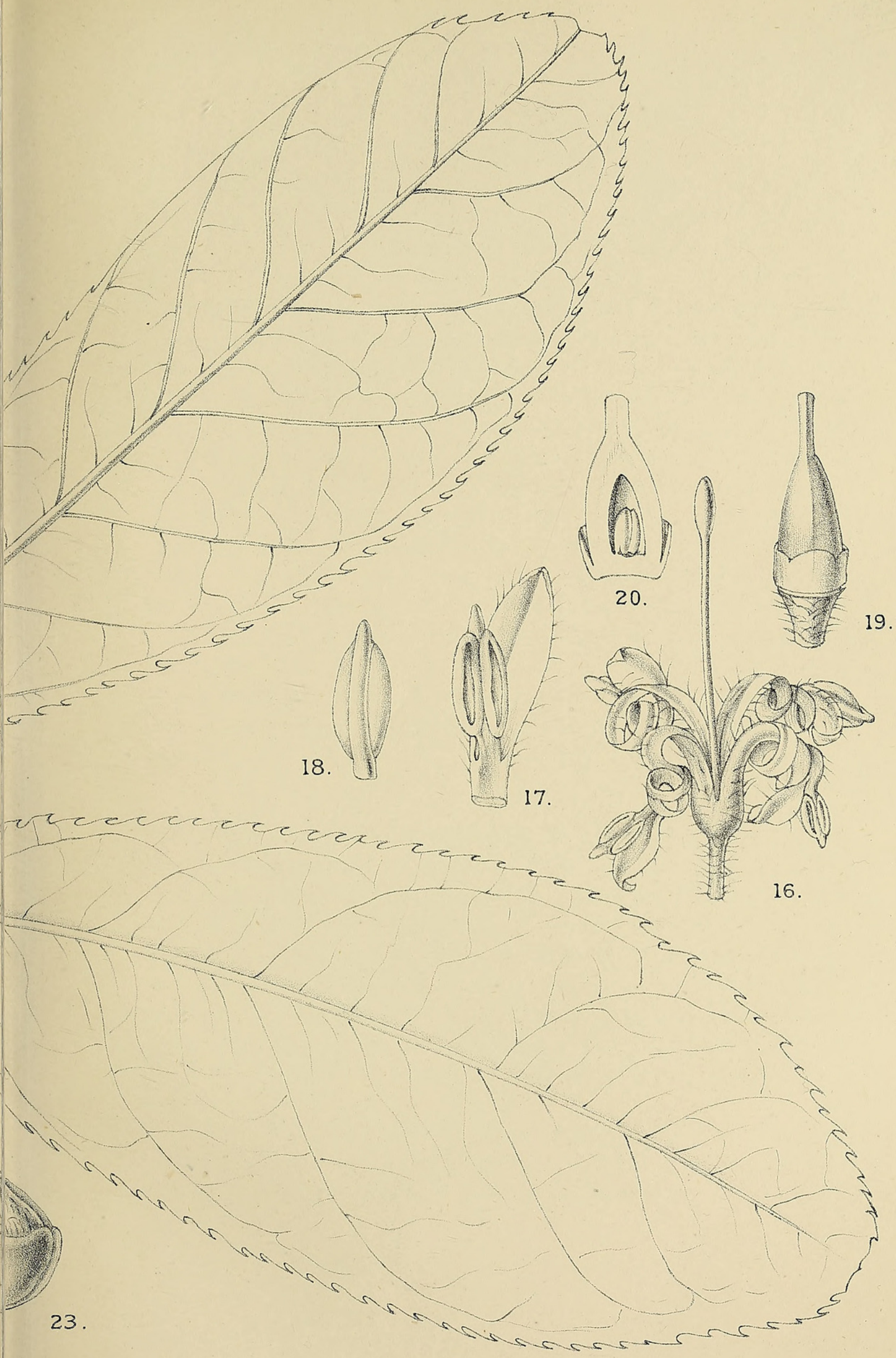

University Press, Oxford. 


\section{$2 \mathrm{BHL}$ Biodiversity Heritage Library}

Hemsley, W. Botting. 1895. "Descriptions of some new plants from Eastern Asia, chiefly from the island of Formosa, presented by Dr. Augustine Henry, F.L.S., to the Herbarium, Royal Gardens, Kew." Annals of botany 9, 143-160. https://doi.org/10.1093/oxfordjournals.aob.a090727.

View This Item Online: https://www.biodiversitylibrary.org/item/233478

DOI: https://doi.org/10.1093/oxfordjournals.aob.a090727

Permalink: https://www.biodiversitylibrary.org/partpdf/318370

\section{Holding Institution}

Smithsonian Libraries

\section{Sponsored by}

Biodiversity Heritage Library

\section{Copyright \& Reuse}

Copyright Status: Not in copyright. The BHL knows of no copyright restrictions on this item.

This document was created from content at the Biodiversity Heritage Library, the world's largest open access digital library for biodiversity literature and archives. Visit BHL at https://www.biodiversitylibrary.org. 